\title{
Metanephric Mass of the Intermediate Mesoderm
}

National Cancer Institute

\section{Source}

National Cancer Institute. Metanephric Mass of the Intermediate Mesoderm. NCI

Thesaurus. Code C34208.

A mass of cells located on the caudal part of the intermediate mesoderm that will develop into the nephrons of the kidney, and, together with the ureteric bud, will give rise to the permanent kidney. 